\title{
Meloxicam-induced enteropathy of the small bowel
}

\author{
Chaitanya Are MBBS, Mohit Turagam MBBS, John A. Aucar MD MSHI, Eugene Greenberg MD
}

A 52-year-old woman was referred to the gastroenterology clinic for evaluation of abdominal pain and a change in her bowel habits. The patient reported an eight-week history of colicky postprandial pain in her right lower quadrant that did not radiate and episodic vomiting in association with the pain. No relieving factors were noted. She also reported newonset constipation with no other constitutional symptoms. Her medical history included hiatus hernia with reflux symptoms, obesity, dietcontrolled diabetes mellitus, hypertension, osteoarthritis and a remote history of endometriosis. Her surgical history consisted of a cholecystectomy and total abdominal hysterectomy with bilateral salpingo-oophorectomy. She had no family history of inflammatory bowel disease or cancer of the gastrointestinal tract. She was taking omeprazole $(20 \mathrm{mg} / \mathrm{d})$, lisinopril $(20 \mathrm{mg} / \mathrm{d})$ and meloxicam $(15 \mathrm{mg} / \mathrm{d})$. She had been taking the meloxicam for several years for osteoarthritis. A screening colonoscopy performed two years earlier was normal.

On physical examination, the patient's abdomen was soft, with no tenderness or palpable organomegaly. Results of a digital rectal examination and a stool guaiac test were normal. Laboratory data showed normal blood urea nitrogen and creatinine levels and a hemoglobin level of 108 (normal 110-160) g/L, which reflected a drop of $40 \mathrm{~g}$ over the preceding eight months. Iron deficiency was present, as reflected by a serum iron level of 2.6 (normal 9-27) $\mu \mathrm{mol} / \mathrm{L}$, a ferritin level of 5.2 (normal 10-200) $\mu \mathrm{g} / \mathrm{L}$ and a total iron-binding capacity of 89.5 (normal 4566) $\mu \mathrm{mol} / \mathrm{L}$. Her serum albumin level was 29 (normal 32-55) g/L with normal levels of serum transaminases. A contrast-enhanced computed tomography scan of the abdomen showed no structural abnormality or inflammatory process. Endoscopy of the upper gastrointestinal tract and flexible sigmoidoscopy showed normal mucosa. Video capsule endoscopy showed multiple areas of ulceration, bleeding and diaphragm-like strictures in the jejunum and ileum (Figure 1). Based on these findings, enteropathy induced by non- steroidal anti-inflammatory drugs (NSAIDs) was suspected and the meloxicam stopped.

At follow-up three months later, the patient reported substantial alleviation of her abdominal pain, and there was no further drop in the hemoglobin level. A repeat video capsule endoscopy showed healing of the bleeding ulcers. Based on these findings and the improvement in her condition following the discontinuation of meloxicam, we concluded that our patient had NSAIDinduced enteropathy of the small bowel secondary to meloxicam use. At follow-up six months later, she was asymptomatic and had a hemoglobin level of $126 \mathrm{~g} / \mathrm{L}$.

\section{Discussion}

NSAIDs are one of the most commonly used groups of medications worldwide. Apart from the well-documented toxicity to the upper gastrointestinal tract, these medications are now known to cause serious enteropathy of the small bowel. NSAID-induced enteropathy is frequently overlooked by clinicians because of its vague presentation. Up to $70 \%$ of patients taking NSAIDs long term may have asymptomatic enteropathy of the small bowel. ${ }^{1}$

\section{Pathogenesis}

NSAID-induced enteropathy appears to be a multi-stage process involving increased intestinal permeability, loss of function in the intestinal barrier resulting in bacterial invasion, and an

\section{- KEY POINTS}

- Up to $70 \%$ of patients taking nonsteroidal anti-inflammatory drugs (NSAIDs) may have asymptomatic enteropathy.

- Small-bowel lesions (mucosal tears and ulcers) from NSAID-induced enteropathy are an important cause of occult gastrointestinal blood loss.

- The formation of diaphragm-like strictures in the small bowel should be considered in the differential diagnosis of otherwise unexplained chronic abdominal pain and intestinal obstruction in patients taking NSAIDs.

- The mainstay of treatment is to stop NSAID use. 
inflammatory tissue reaction. ${ }^{1}$ NSAIDs uncouple mitochondrial oxidative phosphorylation, which reduces the intracellular levels of adenosine triphosphate in the enterocyte. This in turn reduces the integrity of intercellular junctions and increases intestinal permeability. As a result of the increased permeability, intestinal mucosa is susceptible to damage from luminal contents such as bile acids, hydrolytic and proteolytic enzymes, and bacteria. The damage to the mucosa triggers an inflammatory response, with production of inflammatory cytokines and neutrophil infiltration. Release of lysosomal enzymes from neutrophils results in tissue damage in the form of ulceration. The development of ulcers and the subsequent reparative process is thought to result in diaphragm-like strictures. ${ }^{1,2}$

Prostaglandin synthesis from arachidonic acid by the damaged cells is decreased secondary to the inhibition of cyclo-oxygenase (COX) by NSAIDs. This effect further increases the damage caused by the inflammatory response. Recent studies have shown that both isoforms of the COX enzyme (COX-1 and COX-2) play a role in maintaining mucosal integrity of the small intestine. ${ }^{3}$ The microcirculatory dysfunction and increase in intestinal motility noted with the use of NSAIDs may also play a contributory role in the development of NSAID-induced enteropathy. ${ }^{3}$

\section{Complications}

NSAID-induced enteropathy often goes undetected because patients are asymptomatic. Increased intestinal permeability has been reported to occur as early as 12 hours after a single dose of most conventional NSAIDs. ${ }^{2}$ The spectrum of NSAID-induced enteropathy is summarized in Box 1.

How long clinically significant disease takes to develop is unclear. Protein loss due to enteropathy can result in clinically significant hypoalbuminemia. Chronic occult blood loss from mucosal tears and ulcerations can lead to iron deficiency anemia, as noted in our patient. The diaphragm-like strictures that form after the ulcers heal may cause patients to experience nausea, vomiting, colicky abdominal pain, intestinal obstruction and even perforation.

\section{Diagnosis}

The diagnosis of NSAID-induced enteropathy requires awareness of the potential toxic effects of NSAIDs. Often endoscopy of the upper gastrointestinal tract and colonoscopy do not show abnormal mucosal findings because the lesions may be predominantly located in the small intestine. Factors that help establish the diagnosis include typical endoscopic findings of erosions, tears, ulcers or typical diaphragm-like strictures, along with improvement in clinical and endoscopic findings after the NSAID is stopped.

Video capsule endoscopy and double-balloon endoscopy supplement endoscopy of the upper and lower gastrointestinal tracts in visualizing the small bowel. Direct visualization helps to rule out other causes such as inflammatory bowel disease and cancer. The diagnostic yield of video capsule endoscopy and double-balloon endoscopy in the evaluation of the small bowel for occult bleeding, inflammatory findings and tumour detection was not found to differ substantially. ${ }^{4}$ However, cost-effectiveness favours video capsule endoscopy over push enteroscopy and radiologic studies in the diagnosis of obscure bleeding. ${ }^{4}$

The potential complications of video capsule endoscopy are retention of the capsule and obstruction, especially in the presence of strictures. The complications could be reduced with
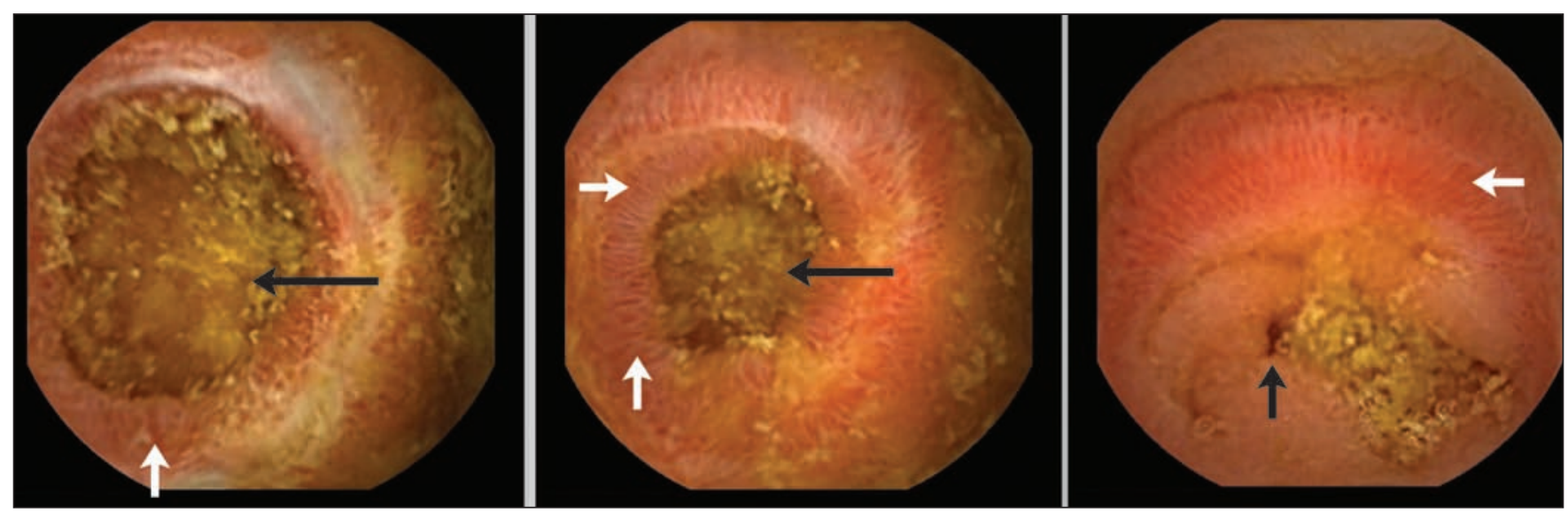

Figure 1: Images from video capsule endoscopy of the small bowel in a 52-year-old woman with meloxicam-induced enteropathy, showing inflammation and erosion of mucosa (white arrows) and diaphragm-like strictures resulting in various degrees of luminal narrowing (black arrows). 
use of the patency capsule. The patency capsule is the same size as the video capsule and consists of a small radiofrequency identification tag enclosed in a lactose capsule. When retained in the intestine for more than 30 hours, the lactose capsule disintegrates, leaving behind the tag. Patency of the intestinal lumen is determined by passage of an intact capsule or the absence of a signal from the tag in the body after 30 hours. ${ }^{4}$

Radiologic studies can help establish a diagnosis, but they often yield false-negative results. The diaphragm-like strictures may be misinterpreted as exaggerated plicae circulares, and contrast studies are not sensitive for detecting mucosal lesions.

Other tests include the indium-labelled leukocyte technique and single-stool fecal calprotectin excretion. Even though these tests have been validated for identifying inflammation associated with NSAID-induced enteropathy, they are limited by their lack of specificity. ${ }^{2}$

\section{Prevention}

Effective strategies to prevent NSAID-induced enteropathy are not available. The results of prophylaxis with misoprostol have been variable and dose dependent, with high doses associated with significant gastrointestinal side effects. ${ }^{5}$ Reduction in gastric acid secretion does not play

\section{Box 1: Spectrum of NSAID-induced enteropathy ${ }^{1,2}$}

Small bowel

- Increased intestinal permeability

- Intestinal inflammation

- Malabsorption and mucosal atrophy

- Protein-losing enteropathy

- Ulcers

- Intestinal perforation

- Diaphragm-like strictures

- Villous atrophy

Colon and rectum

- Nonspecific colitis

- Eosinophilic colitis

- Collagenous colitis

- Colonic ulcers

- Diaphragm-like strictures

- Proctitis

- Rectal ulcers

- Effects on pre-existing disease

- Severe complications of diverticular disease (perforation, bleeding)

- Relapse of inflammatory bowel disease

- Bleeding from angiodysplastic lesions

Note: NSAID = nonsteroidal anti-inflammatory drug. an important role in prevention. ${ }^{1}$ As noted in our patient, concurrent use of omeprazole did not prevent the NSAID-induced enteropathy.

Rebamipide, a new mucosal protective agent not currently available in Canada or the United States, enhances mucosal defence by scavenging free radicals and temporarily activating genes encoding the COX-2 enzyme. In a study involving healthy volunteers, rebamipide was found to be significantly more efficacious than placebo in preventing NSAID-induced injury to the mucosa of the small intestine. ${ }^{6}$

\section{Treatment}

The mainstay of treatment is to stop NSAID use. Switching from one NSAID to another does not offer any benefit, because enteropathy has been reported with most conventional NSAIDs, including acetylsalicylic acid., ${ }^{2,7}$

Despite an initial report that COX-2 inhibitors produced no change in intestinal permeability in healthy volunteers, ${ }^{8}$ a recent study noted smallbowel injury in up to $50 \%$ of patients taking COX2-selective agents for three months or longer. ${ }^{9}$ No statistically significant difference in the prevalence of small-bowel injury was noted between patients taking COX-2-selective agents and those taking conventional NSAIDs. ${ }^{9}$

Even though long-term concurrent use of misoprostol may not prevent the development of enteropathy, it does reduce intestinal inflammation in patients with established NSAIDinduced enteropathy and lessens the severity of the disease.

The reduction in intestinal inflammation and blood loss reported with the use of metronidazole has been attributed to the antibacterial properties of the drug and the role of bacterial invasion in NSAID-induced enteropathy. ${ }^{10}$ Another probable mechanism underlying the protective effect of metronidazole is the reduction in the degree of uncoupling of mitochondrial oxidative phosphorylation induced by NSAIDs.

Sulfasalazine is another drug reported to reduce intestinal inflammation and blood loss..$^{1,2}$ However, these results were from small pilot studies and require careful interpretation.

Although the mucosal erosions and ulcers improve after NSAIDs are stopped, the effect on the diaphragm-like strictures is unclear. When symptoms persist despite medical therapy, surgery may be necessary, and the diagnosis is often made at laparotomy.

\section{Conclusion}

Patients with NSAID-induced enteropathy often have multiple comorbidities and are taking mul- 
tiple medications. Unfamiliarity with the condition can result in delayed diagnosis and increased morbidity. NSAID-induced enteropathy should be considered in the differential diagnosis of anemia, abdominal pain and intestinal obstruction in patients taking NSAIDs.

\section{References}

1. Bjarnason I, Takeuchi K. Intestinal permeability in the pathogenesis of NSAID-induced enteropathy. J Gastroenterol 2009; 44(Suppl 19):23-9.

2. Lanas A, Sopeña F. Nonsteroidal anti-inflammatory drugs and lower gastrointestinal complications. Gastroenterol Clin North Am 2009;38:333-52

3. Higuchi K, Umegaki E, Watanabe T, et al. Present status and strategy of NSAIDs-induced small bowel injury. J Gastroenterol 2009; 44:879-88.

4. Semrad CE. Small bowel enteroscopy: territory conquered, future horizons. Curr Opin Gastroenterol 2009;25:110-5.

5. Davies GR, Wilkie ME, Rampton DS. Effects of metronidazole and misoprostol on indomethacin-induced changes in intestinal permeability. Dig Dis Sci 1993;38:417-25.

6. Niwa Y, Nakamura M, Ohmiya N, et al. Efficacy of rebamipide for diclofenac-induced small-intestinal mucosal injuries in healthy subjects: a prospective, randomized, double-blinded, placebo-controlled, cross-over study. J Gastroenterol 2008;43: 270-6.

7. Endo H, Hosono $\mathrm{K}$, Inamori M, et al. Characteristics of small bowel injury in symptomatic chronic low-dose aspirin users: the experience of two medical centers in capsule endoscopy. J Gastroenterol 2009;44:544-9.

8. Sigthorsson G, Crane R, Simon T, et al. COX-2 inhibition with rofecoxib does not increase intestinal permeability in healthy subjects: a double blind crossover study comparing rofecoxib with placebo and indomethacin. Gut 2000;47:527-32.

9. Maiden L, Thjodleifsson B, Seigal A, et al. Long-term effects of nonsteroidal anti-inflammatory drugs and cyclooxygenase- 2 selective agents on the small bowel: a cross-sectional capsule enteroscopy study. Clin Gastroenterol Hepatol 2007;5:1040-5.

10. Bjarnason I, Hayllar J, Smethurst P, et al. Metronidazole reduces inflammation and blood loss in NSAID enteropathy. Gut 1992; 33:1204-8.

Affiliations: From the Departments of Internal Medicine (Are, Turagam, Greenberg) and Surgery (Aucar), University of Illinois at Urbana-Champaign; and the Carle Physician Group (Aucar, Greenberg), Urbana, Ill.

Contributors: Chaitanya Are and Mohit Turagam drafted the manuscript. All of the authors critically revised the article for important intellectual content and approved the final version submitted for publication.

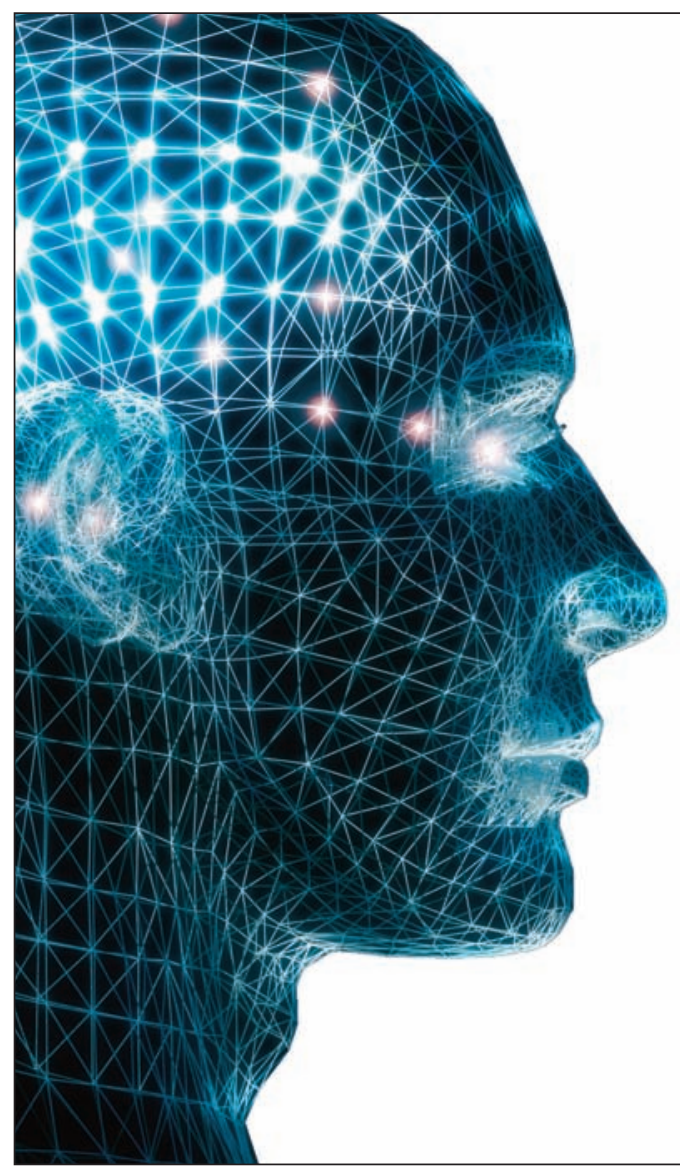

\title{
Be one of the first to know!
}

\author{
And sign up for Weekly Alerts of new content \\ posted on cmaj.ca \\ Don't miss out on online-only news and research \\ articles that you won't see in print.
}

Go to www.cmaj.ca/cgi/alerts/etoc to sign up!

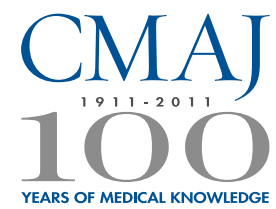

